Review

\title{
Apelin/APJ system: A key therapeutic target for liver disease
} \author{
Shuang-Yu Lv ${ }^{1}$, Binbin Cui ${ }^{1}$, Wei-Dong Chen ${ }^{1,3}$ and Yan-Dong Wang ${ }^{2}$ \\ ${ }^{1}$ Key Laboratory of Receptors-Mediated Gene Regulation and Drug Discovery, School of Medicine, Henan University, Kaifeng, \\ Henan, P. R. China \\ ${ }^{2}$ State Key Laboratory of Chemical Resource Engineering, College of Life Science and Technology, Beijing University of \\ Chemical Technology, Beijing, P. R. China \\ ${ }^{3}$ Key Laboratory of Molecular Pathology, School of Basic Medical Science, Inner Mongolia Medical University, Hohhot, Inner \\ Mongolia, P. R. China \\ Correspondence to: Yan-Dong Wang, email: ydwangbuct2009@163.com \\ Wei-Dong Chen, email: wdchen666@163.com \\ Keywords: apelin; APJ; liver; cirrhosis; fibrosis
}

Received: April 19, $2017 \quad$ Accepted: November 11, $2017 \quad$ Published: December 01, 2017

Copyright: Lv et al. This is an open-access article distributed under the terms of the Creative Commons Attribution License 3.0 (CC BY 3.0), which permits unrestricted use, distribution, and reproduction in any medium, provided the original author and source are credited.

\section{ABSTRACT}

Apelin, a new bioactive peptide, was identified as an endogenous ligand for APJ (Angiotensin II receptor-like 1). Apelin and its receptor have an abundant distribution in central nervous system and peripheral tissues, including liver. Apelin/APJ has diverse physiological and pathological effects, including regulation of cardiovascular function, angiogenesis, fluid homeostasis and so on. Apelin/APJ system may act as a novel potential therapeutic target for liver disease. In this article, we review the role of apelin/APJ system in liver fibrosis, hepatitis, hepatic cirrhosis, liver injury and metabolic liver disease.

\section{INTRODUCTION}

APJ (Angiotensin II receptor-like 1) was discovered by O'Dowd in 1993 [1]. The gene encoding APJ is intronless in the coding region and its approved gene symbol is $A P L N R$ in human and Aplnr in the mouse and the rat [2]. In human, $A P L N R$, located on chromosome $11 q 12$, encodes a 380 -amino acid protein $[1,2]$. In the mouse and the rat, Aplnr, present at the chromosomes 2E1 and 3q24, respectively, encodes 377-amino acid proteins [3-5]. The human APJ is a G-protein-coupled receptor (GPCR) and the similarities of primary structure of APJ and angiotensin II receptor type 1 (AT1R) is 31\%. However, angiotensin II could not combine with APJ [1].

Apelin, firstly extracted from bovine stomach, was confirmed as an endogenous ligand for APJ [6]. The human apelin gene expresses a 77-amino acid prepropeptide, called preproapelin, and is located on chromosome Xq25-26 [6]. The preproapelin contains a signal peptide at its $\mathrm{N}$-terminal, directing the secretory pathway of apelin. The preproapelin, including several binding sites for endopeptidases to cut out, was processed and generated to different active fragments, such as apelin (66-77), named apelin-12, apelin (65-77), named apelin-13, apelin (61-77), named apelin-17, and apelin (42-77), named apelin-36 [4, 7]. [pGlu]apelin-13, the pyroglutamylated form of apelin-13, has been reported to be protected from exopeptidase degradation [4]. Among the different forms of apelin fragments, apelin-13 and [pGlu]apelin-13 are the most potent activators for apelin receptor expressed in cell lines [6-9].

Apelin and APJ are highly expressed in the central nervous system (CNS) and peripheral tissues, such as adipose tissue, brain, liver, lung, kidney, and the cardiovascular system in both human and rodents [10]. Apelin and APJ are also expressed in adult rat liver [5]. In rat liver, the positive staining of apelin has been detected in the endothelia of the portal and central veins, and the Kupffer cells using immunohistochemical staining [11]. Recently, Principe et al. showed that apelin and APJ have an abundant distribution in hepatic stellate cells (HSCs) and hepatocytes of the rat cirrhotic liver [12]. Furthermore, APJ is also highly expressed in hepatocytes of human cirrhotic liver [13]. Hence, the apelin/APJ system may produce important effects on physiology and pathophysiology of liver function [13]. 
Apelin/APJ has diverse physiological and pathological effects, including regulation of cancer [14], vascular smooth muscle cells (VSMC) proliferation [15], ischemia/reperfusion injury [16], fluid homeostasis [17], cardiovascular function, angiogenesis, and acting as a neuroendocrine modulator of the hypothalamic-pituitary-adrenal (HPA) axis in response to stress in human or animals [2]. Apelin/APJ signaling is important for embryonic angiogenesis and is upregulated during tumor angiogenesis [18]. Apelin promotes proliferation and migration of vascular endothelial cells, and could stimulate vascular sprouting in vivo even in the absence of vascular endothelial growth factor [19].

In the current work, we have reveiwed the latest research progress about the role of apelin/APJ system in liver disease, including liver fibrosis, hepatitis, hepatic cirrhosis, liver injury, metabolic liver disease and fatty liver disease.

\section{APELIN IN LIVER FIBROSIS AND HEPATITIS}

The major feature of patients with chronic liver disease is hepatic architectural disruption. Actually, the liver of these patients undergoes an intense process of tissue remodelling characterized by chronic inflammation, neoangiogenesis and fibrogenesis [20]. In LX-2 cells, a cell line derived from human HSCs, proinflammatory substances induced the expression of apelin gene [21]. In HSCs, the overexpression of APJ was activated by plateletderived growth factor (PDGF) and proinflammatory cytokines, suggesting that APJ might promote vascular remodeling in fibrogenesis [22].

In LX-2 cells, both profibrogenic molecules angiotensin II and endothelin-1 enhanced apelin expression, and apelin could increase the synthesis of collagen-I and platelet-derived growth factor receptor (PDGFR). Moreover, APJ receptor antagonist F13A drastically reduced collagen-I and PDGFR expression stimulated by angiotensin II and endothelin-1 [21]. Apelin was involved in regulating fibrogenic activity induced by angiotensin II and endothelin-1, and apelin would be an essential regulator of fibrogenesis in human liver disease [21]. The roles of apelin/ APJ system in liver fibrosis were shown in Figure 1. In $\mathrm{CCl}_{4}$-treated rats as a fibrosis animal model, F13A reduced hepatic collagen content, improved mean arterial pressure (MAP) and portal pressure (PP), ameliorated cell viability,

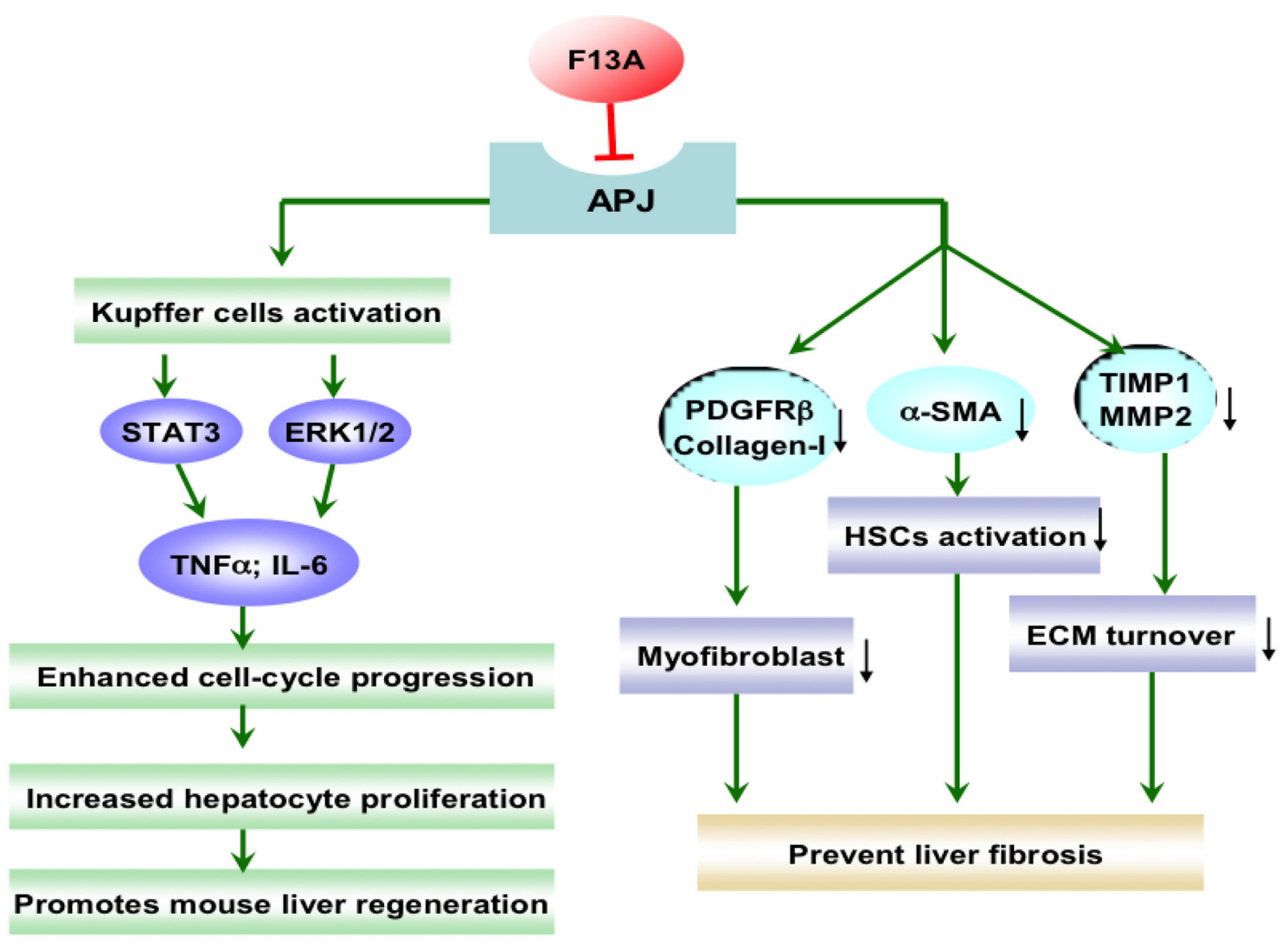

Figure 1: The effect of apelin/APJ system blockade on liver fibrosis and liver regeneration. F13A, apelin-13(F13A); PDGFR $\beta$, platelet-derived growth factor receptor $\beta$; a-SMA, a-smooth muscle actin; TIMP, tissue inhibitor of matrix metalloproteinase; MMP, matrix metalloproteinase; HSCs, hepatic stellate cells; ECM, extracellular matrix; STAT3, signal transducer and activator of transcription 3; ERK, extracellular signal-related kinase; TNF, tumor necrosis factor; IL-6, interleukin-6; $\downarrow$, decrease. Green arrow denotes stimulation. Red arrow denotes suppression. 
and inhibited angiogenesis and cell infiltrate [23]. These effects were associated with reduction of PDGFR $\beta$, a-smooth muscle actin (a-SMA), matrix metalloproteinases, and tissue inhibitors of matrix metalloproteinase [23]. Chen et al. has reported that there is a significant linear correlation between the apelin mRNA level and liver fibrosis, serum total bilirubin and the grade of esophageal varices. The hepatic apelin/ APJ system is activated in the progression of biliary atresia (BA), particularly in end-stage cirrhosis [24]. The expression level of apelin indicates the degree of hepatic fibrosis and esophageal varices, so it could be potentially considered as a prognostic factor for BA patients [24]. Considering the close relationship between apelin expression and profibrogenic factors in HSCs, and the alleviation of fibrogenesis and angiogenesis under the condition of APJ blockade in hepatic fibrosis, the apelin/APJ system might be a promising therapeutic target for liver fibrosis.

Apelin level varies at the different stages of chronic hepatitis $\mathrm{C}(\mathrm{CHC})$, which may lead to fibrosis progression. It was found that TNF- $\alpha$ was negatively correlated to adjusted apelin in CHC patients [25]. Apelin is expressed in the liver of hepatitis-C virus (HCV) patients but not healthy individuals and it is involved in the disease progression [26].

\section{APELIN AND HEPATIC CIRRHOSIS}

The circulating level of apelin was increased in patients with cirrhosis compared with healthy subjects [12]. In addition, apelin protein and gene were upregulated in cirrhotic liver compared with normal liver in humans [27]. Recent clinical study demonstrates that the serum apelin level showed a significant relationship with the severity of liver cirrhosis in patients with chronic liver disease (CLD) [28]. In rats with cirrhosis, the apelin levels were higher than controls, and apelin as well as APJ mRNA also showed an obvious rise in hepatic tissue [12]. Treatment with the F13A, an apelin receptor antagonist, alleviated hepatic fibrosis and vessel density and improved cardiovascular performance in rats with cirrhosis. These results suggest that blocking apelin/APJ signaling pathway might contribute to treatment of cirrhosis and related diseases [12]. In the sinusoid lining cells of human cirrhotic liver, the APJ mRNA and protein expression was upregulated. The highly expressed APJ was detected in activated HSCs, myofibroblasts, and fibroblasts in Child-C cirrhosis liver [22]. These results indicate that APJ might have effect on vascular remodeling and increased portal hypertension in cirrhosis [22]. The apelin/ APJ was activated in patients with cirrhosis and blocking apelin/APJ system could alleviate symptoms of hepatic cirrhosis, suggesting that apelin/APJ system is a potential therapeutic target of hepatic cirrhosis.

In cirrhosis, proliferation of hepatic arterial capillaries leads to an acceleration of arterial blood pouring into the sinusoids, increasing the sinusoidal blood pressure in relation to the defenestration of sinusoidal endothelial cell and consequently leading to the portal hypertension in liver cirrhosis [27, 29]. Apelin was weakly expressed in hepatic sinusoidal endothelial cells and in proliferated arterial capillaries directly connecting to the sinusoids in early stage cirrhotic liver, while apelin was strongly expressed in proliferated arterial capillaries in end stage cirrhosis [27]. Additionally, apelin levels were significantly correlated with the severity of liver disease in patients with alcoholic cirrhosis. Apelin, diponectin and RBP4 levels are deregulated in liver cirrhosis depending on the degree of liver dysfunction [30].

The progression of liver fibrogenesis and cirrhosis is dependent on the different dynamic inflammatory state [31]. With extension and distortion of the normal hepatic architecture of liver fibrosis, tissue hypoxia becomes an essential regulator of the production of proinflammatory and proangiogenic factors [32]. Hypoxia and proinflammatory factors induce the expression of APJ in LX-2 and HepG2 cells. APJ activation stimulates the expression of angiopoietin- 1 in LX-2 cells, which triggers the generation of vascular endothelial growth factor type A and PDGF BB in HepG2 cells. In addition, activation of APJ promotes the HSCs proliferation. The authors proposed that the activation of the apelin system induced by hypoxia and inflammatory factors contributes to angiogenic and fibroproliferative response in chronic liver disease [13].

In addition, apelin and APJ are overexpressed in hepatocellular carcinoma. The apelin/APJ induces tumor arteriogenesis, and they could work as a signal for arteriogenesis in hepatocellular carcinoma [33]. In hepatoma HepG2 cells, apelin promotes autophagy by inducing the phosphorylation of ERK1/2 and upregulating the expression of Beclin1 [34].

\section{APELIN/APJ IN LIVER INJURY AND LIVER REGENERATION}

Yasuzaki et al. found that mRNA levels of apelin and APJ in the liver were increased in mice with fasmediated liver injury [35]. In $\mathrm{APJ}^{-/-}$mice, the liver injury and apoptotic changes were markedly reduced and the obvious activation of JNK was completely abolished after treatment of Jo2, a fas-agonistic antibody, comparing with WT mice, indicating that apelin/APJ signaling may facilitate fas-induced liver injury at least partially via JNK activation [35]. As we know, the JNK activity is also required for the development of liver cancer in carcinogenesis [36]. In hepatic ischemia reperfusion (I/R) injury rats, apelin-13 as well as leptin produced protective effects. Leptin and apelin treatments reduced lipid peroxidation, alleviated histological tissue damage and improved liver function for hepatic I/R injury rats [37]. However, its underlying mechanism remains to be elucidated. 
Liver regeneration is a process of adaptive growth caused by specific stimuli and involves replication of the liver cells, mainly hepatocytes [38]. It is reported that abolishment of apelin/APJ system promotes mouse liver regeneration. Blockade of the apelin/APJ system using F13A promoted hepatocyte proliferation, increasing the secretion of TNF-a and IL-6 by promoting the activation of Kupffer cells during early phase of liver regeneration, showing therapeutic effects in a mouse extended partial hepatectomy (ExPH) model [39] (Figure 1). The apelin/APJ system has an abundant distribution in liver and APJ knock out or abolishment could promote liver regeneration and relieve liver injury. Consequently, the apelin/APJ system would potentially be as a target for the treatment of liver injury and liver regeneration.

\section{APELIN/APJ IN METABOLIC LIVER DISEASES}

Butruille et al. found that the apelin/APJ system is changed mostly in adipose tissue, liver and kidney in obese and insulin-resistant female mice, suggesting that apelin/APJ system may be involved in regulating pathologic status of these tissues under the condition of obesity and diabetes [40]. APJ was detected in HepG2 cells, mouse primary hepatocytes and mouse liver tissue. Apelin reversed the decrease of glycogen level induced by TNF-a, which was mediated by JNK-IRS1-AKT-GSK pathway in HepG2 cells, hepatocytes and liver tissue of mice [41]. This effect was blocked by F13A, suggesting that APJ was involved in the improvement of apelin on the reduction of glycogen synthesis induced by TNF-a [41]. However, hypothalamic apelin could lead to deleterious effect for liver with diabetes. I.c.v. injection with apelin stimulates liver glycogenolysis and gluconeogenesis via the sympathetic nervous system (SNS), inducing peripheral hyperglycemia in normal mice. And the regulation of central apelin on glycemia was dependent on the APJ receptor and the production of hypothalamic reactive oxygen species (ROS) [42] (Figure 2).

Apelin, recognized as an adipokine, was shown to be correlated with nonalcoholic fatty liver disease (NAFLD). Ercin et al. showed that plasma levels of apelin-12 were higher in patients with NAFLD than in healthy comparison subjects [43]. Moreover, the apelin level has a positive correlation with BMI and homeostasis

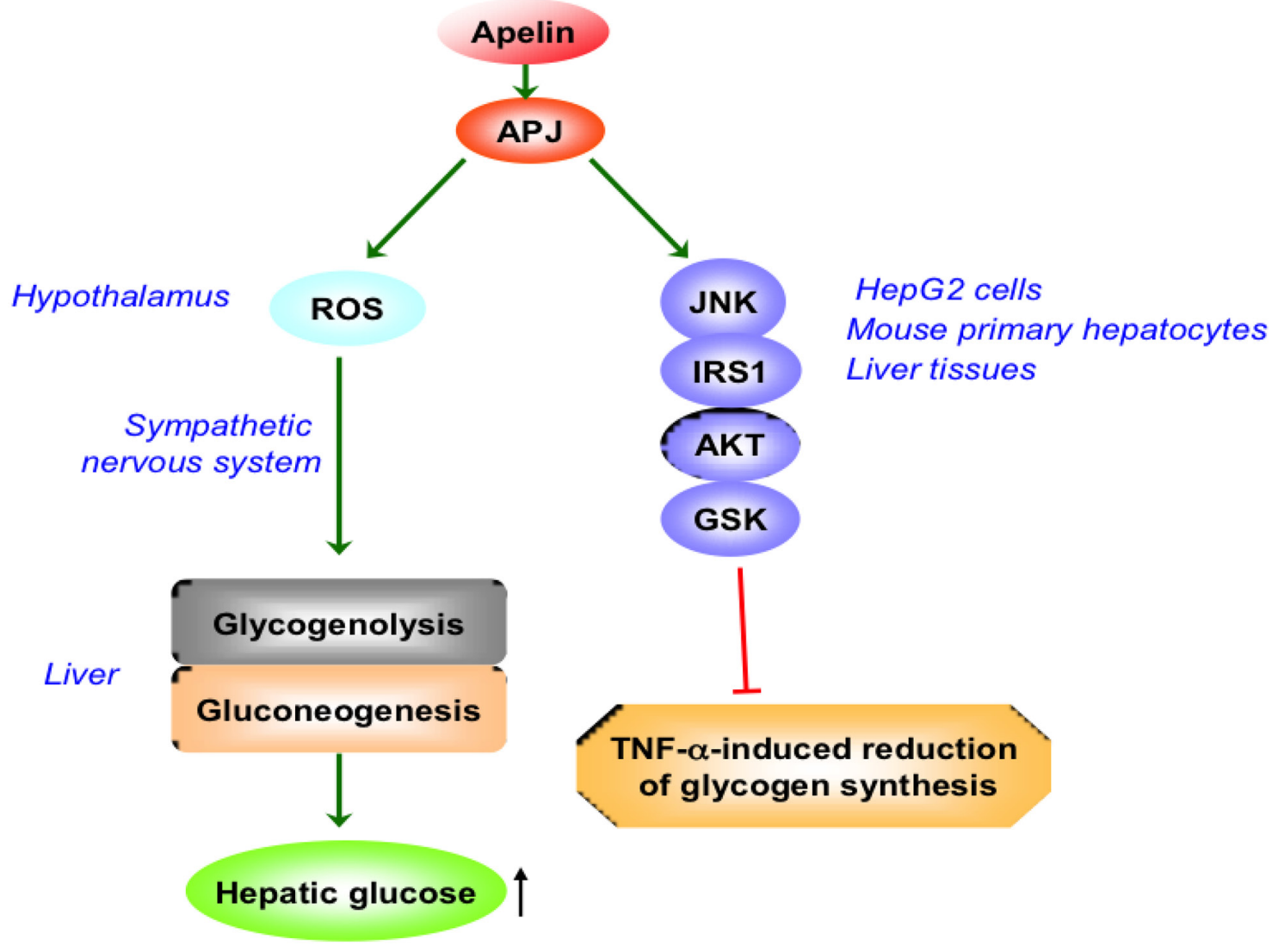

Figure 2: The role of apelin/APJ system on hepatic glucose metabolism. ROS, reactive oxygen species; JNK, c-Jun N-terminal kinase; IRS-1, insulin receptor substrate-1; GSK, glycogen synthase kinase; TNF, tumor necrosis factor; $\uparrow$, increase. Green arrow denotes stimulation. Red arrow denotes suppression. 
model assessment (HOMA) indexes in subjects with NAFLD [43]. Additionally, the serum levels of apelin-36 were markedly higher in NAFLD patients than in healthy individuals [44]. In NAFLD patients, serum apelin-36 levels showed a slight relation with HOMA of insulin resistance [44]. Liver malondialdehyde (MDA) level was significantly correlated with subcutaneous apelin gene expression in rats with high-fat feeding [45].

\section{CONCLUSIONS AND PERSPECTIVES}

In summary, activation of APJ receptor seems to promote development of liver disease, whereas blocking APJ receptor signaling could be used for treatment of liver diseases, such as hepatic cirrhosis and liver injury, and it was beneficial to liver regeneration. Further studies on the mechanism underlying the effect of apelin-APJ signalling in liver disease need to be investigated. Although the experimental evidence supporting the roles for apelin/APJ in liver diseases is overwhelming, the clinical evidence is limited. Further studies were wanted to determine whether these agents could be used in humans undergoing liver disease or surgery. In addition, development of the more effective antagonists and the more stable nonpeptidic APJ antagonists might provide us a new therapeutic tool for liver disease in the future.

\section{ACKNOWLEDGMENTS \& GRANT SUPPORT}

This study was supported by the National Natural Science Foundation of China (Grant No. 81472232 and Grant No. 81270522) to WDC, the National Natural Science Foundation of China (Grant No. 81370537 and Grant No. 81672433) to YDW, Program for Science \& Technology Innovation Talents in Universities of Henan Province (HASTIT, Grant No. 13HASTIT024) and Plan for Scientific Innovation Talent of Henan Province to WDC, and the National Natural Science Foundation of China (Grant No. 81600974), the Key Scientific Research Program for Universities of Henan Province (Grant No. 17A310003) and the Scientific Research Foundation of Henan University (Grant No. 2015YBZR050) to SYL.

\section{CONFLICTS OF INTEREST}

The authors have no conflicts of interest to declare.

\section{REFERENCES}

1. O'Dowd BF, Heiber M, Chan A, Heng HH, Tsui LC, Kennedy JL, Shi X, Petronis A, George SR, Nguyen T. A human gene that shows identity with the gene encoding the angiotensin receptor is located on chromosome 11. Gene. 1993; 136:355-60. https://doi. org/10.1016/0378-1119(93)90495-O.
2. O'Carroll AM, Lolait SJ, Harris LE, Pope GR. The apelin receptor APJ: journey from an orphan to a multifaceted regulator of homeostasis. J Endocrinol. 2013; 219:R13-35. https://doi.org/10.1530/JOE-13-0227.

3. Devic E, Paquereau L, Vernier P, Knibiehler B, Audigier Y. Expression of a new $G$ protein-coupled receptor $\mathrm{X}-\mathrm{msr}$ is associated with an endothelial lineage in Xenopus laevis. Mech Dev. 1996; 59:129-40. https://doi. org/10.1016/0925-4773(96)00585-0.

4. Hosoya M, Kawamata Y, Fukusumi S, Fujii R, Habata Y, Hinuma S, Kitada C, Honda S, Kurokawa T, Onda $\mathrm{H}$, Nishimura O, Fujino M. Molecular and functional characteristics of APJ. Tissue distribution of mRNA and interaction with the endogenous ligand apelin. J Biol Chem. 2000; 275:21061-67. https://doi.org/10.1074/jbc. M908417199.

5. O'Carroll AM, Selby TL, Palkovits M, Lolait SJ. Distribution of mRNA encoding B78/apj, the rat homologue of the human APJ receptor, and its endogenous ligand apelin in brain and peripheral tissues. Biochim Biophys Acta. 2000; 1492:72-80. https://doi.org/10.1016/ S0167-4781(00)00072-5.

6. Tatemoto K, Hosoya M, Habata Y, Fujii R, Kakegawa T, Zou MX, Kawamata Y, Fukusumi S, Hinuma S, Kitada C, Kurokawa T, Onda H, Fujino M. Isolation and characterization of a novel endogenous peptide ligand for the human APJ receptor. Biochem Biophys Res Commun. 1998; 251:471-76. https://doi.org/10.1006/bbrc.1998.9489.

7. Kawamata Y, Habata Y, Fukusumi S, Hosoya M, Fujii R, Hinuma S, Nishizawa N, Kitada C, Onda H, Nishimura O, Fujino M. Molecular properties of apelin: tissue distribution and receptor binding. Biochim Biophys Acta. 2001; 1538:162-71. https://doi.org/10.1016/ S0167-4889(00)00143-9.

8. Habata Y, Fujii R, Hosoya M, Fukusumi S, Kawamata Y, Hinuma S, Kitada C, Nishizawa N, Murosaki S, Kurokawa T, Onda H, Tatemoto K, Fujino M. Apelin, the natural ligand of the orphan receptor APJ, is abundantly secreted in the colostrum. Biochim Biophys Acta. 1999; 1452:25-35. https://doi.org/10.1016/S0167-4889(99)00114-7.

9. Medhurst $\mathrm{AD}$, Jennings $\mathrm{CA}$, Robbins MJ, Davis RP, Ellis C, Winborn KY, Lawrie KW, Hervieu G, Riley G, Bolaky JE, Herrity NC, Murdock P, Darker JG. Pharmacological and immunohistochemical characterization of the APJ receptor and its endogenous ligand apelin. J Neurochem. 2003; 84:1162-72. https://doi. org/10.1046/j.1471-4159.2003.01587.x.

10. Kleinz MJ, Davenport AP. Emerging roles of apelin in biology and medicine. Pharmacol Ther. 2005; 107:198-211. https://doi.org/10.1016/j.pharmthera.2005.04.001.

11. Tatemoto K, Takayama K, Zou MX, Kumaki I, Zhang W, Kumano K, Fujimiya M. The novel peptide apelin lowers blood pressure via a nitric oxide-dependent mechanism. Regul Pept. 2001; 99:87-92. https://doi.org/10.1016/ S0167-0115(01)00236-1. 
12. Principe A, Melgar-Lesmes P, Fernández-Varo G, del Arbol LR, Ros J, Morales-Ruiz M, Bernardi M, Arroyo V, Jiménez W. The hepatic apelin system: a new therapeutic target for liver disease. Hepatology. 2008; 48:1193-201. https://doi. org/10.1002/hep.22467.

13. Melgar-Lesmes P, Pauta M, Reichenbach V, Casals G, Ros J, Bataller R, Morales-Ruiz M, Jiménez W. Hypoxia and proinflammatory factors upregulate apelin receptor expression in human stellate cells and hepatocytes. Gut. 2011; 60:1404-11. https://doi.org/10.1136/ gut.2010.234690.

14. Yang Y, Lv SY, Ye W, Zhang L. Apelin/APJ system and cancer. Clin Chim Acta. 2016; 457:112-16. https://doi. org/10.1016/j.cca.2016.04.001.

15. Lv XR, Zheng B, Li SY, Han AL, Wang C, Shi JH, Zhang XH, Liu Y, Li YH, Wen JK. Synthetic retinoid Am80 up-regulates apelin expression by promoting interaction of RAR $\alpha$ with KLF5 and Sp1 in vascular smooth muscle cells. Biochem J. 2013; 456:35-46. https://doi.org/10.1042/ BJ20130418.

16. Yang Y, Lv SY, Lyu SK, Wu D, Chen Q. The protective effect of apelin on ischemia/reperfusion injury. Peptides. 2015; 63:43-46. https://doi.org/10.1016/j. peptides.2014.11.001.

17. Lv SY, Yang YJ, Chen Q. Regulation of feeding behavior, gastrointestinal function and fluid homeostasis by apelin. Peptides. 2013; 44:87-92. https://doi.org/10.1016/j. peptides.2013.03.024.

18. Kälin RE, Kretz MP, Meyer AM, Kispert A, Heppner FL, Brändli AW. Paracrine and autocrine mechanisms of apelin signaling govern embryonic and tumor angiogenesis. Dev Biol. 2007; 305:599-614. https://doi.org/10.1016/j. ydbio.2007.03.004.

19. Cox CM, D’Agostino SL, Miller MK, Heimark RL, Krieg PA. Apelin, the ligand for the endothelial G-protein-coupled receptor, APJ, is a potent angiogenic factor required for normal vascular development of the frog embryo. Dev Biol. 2006; 296:177-89. https://doi.org/10.1016/j. ydbio.2006.04.452

20. Friedman SL. Hepatic stellate cells: protean, multifunctional, and enigmatic cells of the liver. Physiol Rev. 2008; 88:125-72. https://doi.org/10.1152/ physrev.00013.2007.

21. Melgar-Lesmes P, Casals G, Pauta M, Ros J, Reichenbach V, Bataller R, Morales-Ruiz M, Jimenez W. Apelin mediates the induction of profibrogenic genes in human hepatic stellate cells. Endocrinology. 2010; 151:5306-14. https:// doi.org/10.1210/en.2010-0754.

22. Yokomori H, Oda M, Yoshimura K, Machida S, Kaneko F, Hibi T. Overexpression of apelin receptor (APJ/AGTRL1) on hepatic stellate cells and sinusoidal angiogenesis in human cirrhotic liver. J Gastroenterol. 2011; 46:222-31. https://doi.org/10.1007/s00535-010-0296-3.
23. Reichenbach V, Ros J, Fernández-Varo G, Casals G, Melgar-Lesmes P, Campos T, Makriyannis A, Morales-Ruiz M, Jiménez W. Prevention of fibrosis progression in CCl4treated rats: role of the hepatic endocannabinoid and apelin systems. J Pharmacol Exp Ther. 2012; 340:629-37. https:// doi.org/10.1124/jpet.111.188078.

24. Chen W, Oue T, Ueno T, Uehara S, Usui N, Fukuzawa $\mathrm{M}$. Apelin is a marker of the progression of liver fibrosis and portal hypertension in patients with biliary atresia. Pediatr Surg Int. 2013; 29:79-85. https://doi.org/10.1007/ s00383-012-3210-7.

25. El-Mesallamy HO, Hamdy NM, Rizk HH, El-Zayadi AR. Apelin serum level in Egyptian patients with chronic hepatitis C. Mediators Inflamm. 2011; 2011:703031. https:// doi.org/10.1155/2011/703031.

26. Farid RM, Abu-Zeid RM, El-Tawil A. Emerging role of adipokine apelin in hepatic remodelling and initiation of carcinogensis in chronic hepatitis C patients. Int J Clin Exp Pathol. 2014; 7:2707-17.

27. Yokomori H, Oda M, Yoshimura $\mathrm{K}$, Hibi $\mathrm{T}$. Enhanced expressions of apelin on proliferative hepatic arterial capillaries in human cirrhotic liver. Hepatol Res. 2012; 42:508-14. https://doi. org/10.1111/j.1872-034X.2011.00945.x.

28. Lim YL, Choi E, Jang YO, Cho YZ, Kang YS, Baik SK, Kwon SO, Kim MY. Clinical implications of the serum apelin level on portal hypertension and prognosis of liver cirrhosis. Gut Liver. 2016; 10:109-16. https://doi. org/10.5009/gnl14345.

29. Oda M, Azuma T, Nishizaki Y, Kaneko K, Komatsu H, Tsukada N, Watanabe N, Nakamura M, Tsuchiya M. Alterations of hepatic sinusoids in liver cirrhosis: their involvement in the pathogenesis of portal hypertension. $\mathrm{J}$ Gastroenterol Hepatol. 1989; 4:111-13.

30. Kalafateli M, Triantos C, Tsochatzis E, Michalaki M, Koutroumpakis E, Thomopoulos K, Kyriazopoulou V, Jelastopulu E, Burroughs A, Lambropoulou-Karatza C, Nikolopoulou V. Adipokines levels are associated with the severity of liver disease in patients with alcoholic cirrhosis. World J Gastroenterol. 2015; 21:3020-29. https://doi. org/10.3748/wjg.v21.i10.3020.

31. Friedman SL. Molecular regulation of hepatic fibrosis, an integrated cellular response to tissue injury. J Biol Chem. 2000; 275:2247-50. https://doi.org/10.1074/jbc.275.4.2247.

32. Tugues S, Fernandez-Varo G, Muñoz-Luque J, Ros J, Arroyo V, Rodés J, Friedman SL, Carmeliet P, Jiménez W, Morales-Ruiz M. Antiangiogenic treatment with sunitinib ameliorates inflammatory infiltrate, fibrosis, and portal pressure in cirrhotic rats. Hepatology. 2007; 46:1919-26. https://doi.org/10.1002/hep.21921.

33. Muto J, Shirabe K, Yoshizumi T, Ikegami T, Aishima S, Ishigami K, Yonemitsu Y, Ikeda T, Soejima Y, Maehara Y. The apelin-APJ system induces tumor arteriogenesis 
in hepatocellular carcinoma. Anticancer Res. 2014; 34:5313-20.

34. Huang Q, Liu X, Cao C, Lei J, Han D, Chen G, Yu J, Chen L, Lv D, Li Z. Apelin-13 induces autophagy in hepatoma HepG2 cells through ERK1/2 signaling pathwaydependent upregulation of Beclin1. Oncol Lett. 2016; 11:1051-56.

35. Yasuzaki H, Yoshida S, Hashimoto T, Shibata W, Inamori M, Toya Y, Tamura K, Maeda S, Umemura S. Involvement of the apelin receptor APJ in Fas-induced liver injury. Liver Int. 2013; 33:118-26. https://doi.org/10.1111/ liv.12006.

36. Wang YD, Chen WD, Li C, Guo C, Li Y, Qi H, Shen H, Kong J, Long X, Yuan F, Wang X, Huang W. Farnesoid $\mathrm{X}$ receptor antagonizes JNK signaling pathway in liver carcinogenesis by activating SOD3. Mol Endocrinol. 2015; 29:322-31. https://doi.org/10.1210/me.2014-1225.

37. Sagiroglu T, Aksoy MB, Sagiroglu G, Tozkir H, Oguz S, Yalta T, Yagci MA, Sezer A. Effect of leptin and apelin preconditioning on hepatic ischemia reperfusion injury in rats. Indian J Surg. 2014; 76:111-16. https://doi. org/10.1007/s12262-012-0676-4.

38. Chen WD, Wang YD, Meng Z, Zhang L, Huang W. Nuclear bile acid receptor FXR in the hepatic regeneration. Biochim Biophys Acta. 2011; 1812:888-92. https://doi.org/10.1016/j. bbadis.2010.12.006.

39. Yoshiya S, Shirabe K, Imai D, Toshima T, Yamashita YI, Ikegami T, Okano S, Yoshizumi T, Kawanaka H, Maehara Y. Blockade of the apelin-APJ system promotes mouse liver regeneration by activating Kupffer cells after partial hepatectomy. J Gastroenterol. 2015; 50:573-82.
40. Butruille L, Drougard A, Knauf C, Moitrot E, Valet P, Storme L, Deruelle P, Lesage J. The apelinergic system: sexual dimorphism and tissue-specific modulations by obesity and insulin resistance in female mice. Peptides. 2013; 46:94-101. https://doi.org/10.1016/j. peptides.2013.05.013.

41. Chu J, Zhang H, Huang X, Lin Y, Shen T, Chen B, Man Y, Wang S, Li J. Apelin ameliorates TNF- $\alpha$-induced reduction of glycogen synthesis in the hepatocytes through $\mathrm{G}$ proteincoupled receptor APJ. PLoS One. 2013; 8:e57231. https:// doi.org/10.1371/journal.pone.0057231.

42. Drougard A, Duparc T, Brenachot X, Carneiro L, Gouazé A, Fournel A, Geurts L, Cadoudal T, Prats AC, Pénicaud L. Hypothalamic apelin/reactive oxygen species signaling controls hepatic glucose metabolism in the onset of diabetes. Antioxid Redox Signal. 2014; 20:557-573.

43. Ercin CN, Dogru T, Tapan S, Kara M, Haymana C, Karadurmus N, Karslioglu Y, Acikel C. Plasma apelin levels in subjects with nonalcoholic fatty liver disease. Metabolism. 2010; 59:977-81. https://doi.org/10.1016/j. metabol.2009.10.019.

44. Aktas B, Yilmaz Y, Eren F, Yonal O, Kurt R, Alahdab YO, Celikel CA, Ozdogan O, Imeryuz N, Kalayci C, Avsar E. Serum levels of vaspin, obestatin, and apelin-36 in patients with nonalcoholic fatty liver disease. Metabolism. 2011; 60:544-49. https://doi.org/10.1016/j.metabol.2010.05.008.

45. García-Díaz D, Campión J, Milagro FI, Martínez JA. Adiposity dependent apelin gene expression: relationships with oxidative and inflammation markers. Mol Cell Biochem. 2007; 305:87-94. https://doi.org/10.1007/ s11010-007-9531-5. 